\title{
Nonparametric Penalized Spline Model Calibrated Estimator in Complex Survey with Known Auxiliary Information at Both Cluster and Element Levels
}

\author{
Nthiwa Janiffer Mwende ${ }^{1,}$, Ali Salim Islam ${ }^{1}$, Pius Nderitu Kihara ${ }^{2}$ \\ ${ }^{1}$ Department of Mathematics, Egerton University, Nakuru, Kenya \\ ${ }^{2}$ Department of Financial and Actuarial Mathematics, Technical University of Kenya, Nairobi, Kenya \\ Email address: \\ janienthiwa@yahoo.com (N. J. Mwende), asislam54@yahoo.com (A. S. Islam), piuskihara@yahoo.com (P. N. Kihara) \\ ${ }^{*}$ Corresponding author
}

To cite this article:

Nthiwa Janiffer Mwende, Ali Salim Islam, Pius Nderitu Kihara. Nonparametric Penalized Spline Model Calibrated Estimator in Complex Survey with Known Auxiliary Information at Both Cluster and Element Levels. Science Journal of Applied Mathematics and Statistics. Vol. 9, No. 1, 2021, pp. 20-32. doi: 10.11648/j.sjams.20210901.13

Received: November 27, 2020; Accepted: February 16, 2021; Published: March 10, 2021

\begin{abstract}
The present study uses penalized splines (p- spline) to estimate the functional relationship between the survey variable and the auxiliary variable in a complex survey design; where a population divided into clusters is in turn subdivided into strata. This study has considered a case of auxiliary information present at two levels; at both cluster and element levels. The study further applied model calibration technique by penalty function to estimate the population total. The calibration problems at both levels have been treated as optimization problems and solved using penalty functions to derive the estimators for this study. The reasoning behind model calibration is that if the calibration constraints are satisfied by the auxiliary variable, the study expects that the variable of interest's fitted values meets such constraints. This study runs a Monte Carlo simulation to assess the finite sample performance of the penalized spline model calibrated estimator under complex survey data. Simulation studies were conducted to compare the efficiency of p-spline model calibrated estimator with Horvitz Thompson estimator (HT) by mean squared error (MSE) criterion. This study shows that the p-spline model-based estimator is generally more efficient than the HT in terms of the mean squared error. The results have also shown that the estimator obtained is unbiased, consistent and very robust because it does not fail if the model is misspecified for the data.
\end{abstract}

Keywords: Penalized Spline, Nonparametric Model, Auxilliary Information and Optimization Problem

\section{Introduction}

In recent years, Nonparametric estimation methods have gained considerable attention due to their flexibility. One of these methods is the penalized spline estimation. This method requires knowing the number and locations of knots, the degree of the polynomial, and the degrees of freedom. The degrees of freedom are known as the equivalent number of parameters. There are practical rules in the existing literature to determine the degree of the polynomial and the number and locations of knots. The degrees of freedom are established according to the user's experience.

Splines can be classified as regression splines, cubic splines, B-splines, penalized-splines, natural splines, thinplate splines, and smoothing splines, according to De Boor
(2001), [3]. Nonparametric regression using splines has undergone extensive development in recent years. Smoothing splines (Eubank 1988; Wahba 1990) [6, 13] use a knot at each distinct value except the boundary values of the $\mathrm{X}$ variable and control overfitting by applying a rough-ness penalty. Penalized splines (p-splines), formally introduced by Eilers and Marx (1996), [5], are in general computationally inexpensive and allow flexible knot selection yet yield sound performance. P-splines are also easy to implement: there is a close relationship which implies that they can be fitted using widely-available statistical software such as R software and S-Plus (Pinheiro and Bates, 2000), [9] function lme ().

The Horvitz-Thompson (HT) estimator (Horvitz and Thompson 1952), [7] is a standard design-unbiased estimator of population total and weights cases by the inverse of their 
inclusion probabilities (Zhen and little 2003), [15]. This estimator was used in this study as a baseline comparison with p-spline model calibrated estimator.

The concept of auxiliary variables in the present scholarship in statistics denotes independent or predictor variables in a regression analysis. As the name suggests, the variables offer additional information and may be used to improve the estimation of population parameters. Breidt and Opsomer (2000), [1] noted that micro-econometric research is frequently performed using data collected by surveys, which may contain auxiliary information for every unit of the population of interest. As can be expected, many of these surveys use complex sampling plans to reduce costs and increase the estimation efficiency for subgroups of the population. Complex sampling designs result in unequal sampling probabilities for the units in the sample and create data with correlations between observations, violating the assumption that the data are independently and identically distributed (iid) (Sayed, 2010), [12]. This means that the number of population units represented by a given sample unit is not uniform across all of the units in the sample. Although the word complex survey has been used mostly by researchers to refer to different combinations of sampling plans, however, in this research, complex survey refers to a mixture of both stratified and cluster sampling methods (Clair, 2016), [2].

In this study, Nthiwa Janiffer Mwende, et al. (2020), [8] work which considered auxiliary information at cluster level only is extended. This study extends this work to consider auxiliary information available at both the element and cluster levels. The extension is by treating the two levels of calibration problems in a cluster- Strata sampling, as constrained nonlinear optimization problems which is then converted into unconstrained optimization problems. The resulting problems were then solved using the penalty function method to obtain the weights at both cluster and cluster- strata element levels assigned to sample observations from some chi-square distance measures.

This study considered the generalized calibration procedure using model calibration as proposed by $\mathrm{Wu}$ and sitter (2001), [14]. They considered generalized linear and nonparametric regression models for the super population model $\psi$ given in the equation below:

$$
y_{i}=h\left(x_{i}\right)+\varepsilon_{i}
$$

where $\{\varepsilon\}_{i=1}^{n}$ is a sequence of independent and identically distributed random variables with $E\left(\varepsilon_{i}\right)=0$ and $E\left(\varepsilon_{i}^{2}\right)=\sigma^{2}$ and $h\left(x_{i}\right)$ is a smooth function that can be estimated using nonparametric methods like kernel, neural network, and penalized splines. Given $\mathrm{n}$ pair of sample observations $\left(x_{1}, y_{1}\right), \ldots,\left(x_{n}, y_{n}\right)$ from a population of size $N$, of interest,

is the estimator $\hat{h}(x)$ of $h(x)=E_{\psi}(y / x)$. For model calibration, calibration is performed to the population mean of the fitted values $\hat{h}_{i}\left(x_{i}\right)$ (Wu and sitter, 2001), [14]. The study considers a model calibration estimator for the population total $Y_{t}$ given below.

$$
\hat{Y}=\sum_{i \in a} w_{i} y_{i}
$$

Where $a$ is a set of sampled units under a general sampling design while $w_{i}^{\prime}$ s are design weights such that for a given metric, are as close as possible in an average sense to the $z_{i}=\frac{1}{\pi_{i}}$. These weights are obtained by minimizing a given distance measure between the $w_{i}$ 's and $z_{i}$ 's subject to some constraints. The chi-squared distance measure to be minimized is as provided in the equation below

$$
\delta_{a}=\sum_{i \in a} \frac{\left(w_{i}-z_{i}\right)^{2}}{q_{i} z_{i}}
$$

where $q_{i}$ 's are known positive constants uncorrelated with the $z_{i}$ 's, (Deville and Sarndal, 1992), [4] subject to two constraints equation given below

$$
\left\{\begin{array}{l}
\sum_{i \in a} w_{i}=N \\
\sum_{i \in a} w_{i} \hat{h}_{i}=\sum_{i=1}^{N} \hat{h}_{i}
\end{array}\right.
$$

where $\hat{h}_{i}=\hat{h}_{i}\left(x_{i}\right)$.

Model calibration method is intended to provide good efficiency if the model is correctly specified but maintains desirable properties like design consistency if the model is misspecified (Sahar, 2012), [11]. The simulations in this study suggest that for estimation of the finite population total, p-spline model-based predictive estimators are, in general, more efficient than the HT estimator. In situations that favor the HT estimator, the nonparametric model-based estimators are only slightly less efficient.

\section{Fitting of Missing Values}

In this section, the study considered fitting missing values for a population divided into clusters which are then subdivided into strata. This section considered a case where there is auxiliary information known at both cluster and cluster element Levels. The study defined $Q=x_{1}, x_{2}, \ldots, x_{U}$ as a population of auxiliary variables of size $U$ with auxiliary variable; $x_{i}$ being known at $i^{\text {th }}$ cluster and auxiliary variable; $x_{i j k}$ being known at strata elements level. This study considered a population of clusters $\mathrm{G}$ to be partitioned into $\mathrm{C}$ clusters, each of size $M_{i}, i=1,2, \ldots, C$.

Further, each cluster contains $L_{i}$ strata each of size $N_{j}, j=1,2, \ldots, L_{i}$. Let also $y_{i j k}$ be $k^{\text {th }}$ observation in the 
sample from the $j^{\text {th }}$ stratum of $i^{\text {th }}$ cluster and $x_{i}$ be the corresponding auxiliary variable at cluster level. At stage one, a probability sample c of size $m_{i}$ of clusters is drawn from $\mathrm{C}$ according to a fixed design $P_{1}(\bullet)$ (by simple random sampling), where $P_{1}(c)$ is the probability of drawing the sample $\mathrm{c}$ of size $m_{i}$ from $\mathrm{C}$. The first order cluster inclusion probabilities, $\quad P_{1}(\bullet) \quad$ is $\quad \pi_{i}=\operatorname{pr}(i \varepsilon c)=\sum_{i \varepsilon c} P_{1}(c) \quad$ and $\pi_{i, t}=\operatorname{pr}(i, t \varepsilon c)=\sum_{i, t \varepsilon c} P_{1}(c)$. The first and the second order probabilities are the probability of including cluster $i$ in the sample and the probability of including clusters $i$ and $t$ in the sample respectively. At stage two, for every sampled cluster $i \in c$, the study chose a sample $b_{i}$ of elements of size $n_{i}, i=1,2, \ldots, c$, where $n_{i}=n_{i 1}+n_{i 2}+\ldots+n_{i L_{i}}$. Given that $n_{i 1}, n_{i 2}, \ldots, n_{i L_{i}}$ are sample sizes of the sample chosen from $L_{i}$ strata by proportional allocation with inclusion probabilities $\pi_{k / j / i}=p r\left(k, j \varepsilon r_{i} / i \varepsilon c\right)$ and $\pi_{k, p / j / i}=p r\left(k, p \varepsilon r_{i} / i \varepsilon c\right)$. In this case, the first and second order probabilities are the probability of including element $k$ in the sample $b_{i}$ of the $i^{\text {th }}$ cluster and the probability that unit $k$ and $p$ are both included in the sample $b_{i}$ respectively. This study let;

$$
t_{i}=h\left(x_{i}\right)+\varepsilon_{l} ; i=1,2, \ldots, C
$$

to be the ith cluster total, where $\hat{h}\left(x_{i}\right)$ is a smooth function of $x$. Let also $\hat{t}_{c}=\left[\hat{t}_{i}\right]_{i \varepsilon c}$ be the $m_{i}$ dimension vector of $\hat{t}_{i}{ }^{\prime} s$ which is obtained in the sample of clusters.

This study modelled $\hat{h}\left(x_{i}\right)$ in equation (5) by way of penalized spline and performed model calibration on $\hat{h}\left(x_{i}\right)$. Since some information is available at the element level such that for each element in the jth strata of the ith cluster, a nonparametric variable $x_{i j k}$ is available then the first step was to obtain the nonparametric fit for elements; $h_{x_{i j k}}$ using the auxiliary variable $x_{i j k}$ at element level nonparametrically before an estimate of cluster total through stratification for the ith cluster total was found.

Suppose that not all element values of the variable of interest in a given cluster- strata are available and have to be imputed. This study derived a model calibrated estimator of element within cluster as follows;

$$
E\left(y_{i j k}\right)=\hat{h}\left(x_{i j k}\right)
$$

where $\hat{h}\left(x_{i j k}\right)$ and $x_{i j k}$ are defined for every element $k$ in the jth stratum of ith cluster. For simplicity, this study uses $\hat{h}_{i j k}$ for $\hat{h}\left(x_{i j k}\right)$.

Firstly, when penalized splines are used to fit missing values, the present study defined the nonparametric sample fit for elements within clusters, $E_{\psi_{1}}\left(h_{i j k}\right)$ at $x_{i j k}$ due Breidt and Opsomer, (2000) [1] as

$$
\hat{h}_{x_{i j k}}=J_{p s i j k}^{T} y_{i j k}
$$

where $J_{p s i j k}^{T}$ is defined by

$$
J_{p s i j k}^{T}=X_{r i j k}\left(X_{O b}{ }^{T} W_{b} X_{O b}+A \alpha\right)^{-1} X_{O b}{ }^{T} W_{b}
$$

In which a matrix $X_{O}$ is considered with rows

$$
X_{\text {Oijk }}^{T}=\left\{1, x_{i j k}, \ldots, x_{i j k}^{q},\left(x_{i j k}-k_{1}\right)_{+}^{q}, \ldots,\left(x_{i j k}-k_{l}\right)_{+}^{q}\right\}
$$

for $i \varepsilon G, \mathrm{q}$ is the degree of the spline, and the $k_{l}$ are the knots, while $\left(x-k_{1}\right)_{+}=x-k_{1}$ if $x>k_{1}$ and 0 if $x \leq k_{1}$. Further, $X_{O b}$ in equation 8 is the sub matrix of $X_{0}$ which consists of the rows $X_{O i j k}^{T}$ for which the cluster element $k \varepsilon b_{i}, A_{\alpha}=$ $\operatorname{diag}\{0, \ldots, 0, \alpha, \ldots, \alpha\}$ with $\mathrm{q}+1$ zeros on the diagonal followed by $l$ penalty constants $\alpha$.

The study considered the diagonal matrix of inverse inclusion probabilities as;

$$
W=\operatorname{diag}, i \in G\left\{\frac{1}{\pi_{i j k}}\right\} \text { and its sample submatrix in }
$$

equation 8 given as; $W_{b}=\operatorname{diag}, k \in b_{i}\left\{\frac{1}{\pi_{i j k}}\right\}$. As a result, the model calibrated estimator for the sampled cluster total was defined by;

$$
\hat{t}_{i}=\sum_{i \varepsilon C} w_{i j k} \hat{y}_{i j k} .
$$

The optimal weights; $w_{i j k}$ in equation (10) was obtained by the penalty function method. They were obtained by minimizing the chi square distance below as discussed by Deville and Sarndal (1992), [4].

$$
\delta_{c}=\sum_{k \in b_{i}} \frac{\left(w_{i j k}-z_{i j k}\right)^{2}}{q_{i j k} z_{i j k}} .
$$

subject to

$$
\sum_{k \varepsilon b_{i}} w_{i j k}=M_{i}
$$

and

$$
\sum_{k=1}^{n_{i}} w_{i j k} \hat{h}_{i j k}=\sum_{i=1}^{M_{i}} \hat{h}_{i j k}
$$


where $z_{i j k}=\frac{1}{\pi_{i j k}}$ is the inverse of inclusion probability and $q_{i j k}$ are some known positive constants, uncorrelated with $z_{i j k}$. This study followed same optimization procedure by Rao, 1984, [10] and considered an optimization problem of the form Minimize

$$
\begin{aligned}
& \delta_{c}=\sum_{k \in b_{i}} \frac{\left(w_{i j k}-z_{i j k}\right)^{2}}{q_{i j k} z_{i j k}} \text { subject to } \\
& \tau\left(w_{c}, r_{b}, \hat{h}_{i j k}\right)=\sum_{k \varepsilon b_{i}} \frac{\left(w_{i j k}-z_{i j k}\right)^{2}}{q_{i j k} z_{i j k}}+H\left(r_{b}\right)\left(\sum_{k=1}^{n_{i}} w_{i j k} \hat{h}_{i j k}-\sum_{k=1}^{M_{i}} \hat{h}_{i j k}\right)^{2}+H\left(r_{b}\right)\left(\sum_{k=1}^{n_{i}} w_{i j k}-M_{i}\right)^{2} .
\end{aligned}
$$

Differentiating equation (14) partially with respect to $w_{i j k}$ we get

$$
\tau^{1}\left(w_{i j k}, r_{b}, \hat{h}_{i j k}\right)=2 \frac{\left(w_{i j k}-z_{i j k}\right)}{q_{i j k} z_{i j k}}+2 H\left(r_{b}\right) \hat{h}_{i j k}\left(\sum_{k=1}^{n_{i}} w_{i j k} \hat{h}_{i j k}-\sum_{k=1}^{M_{i}} \hat{h}_{i j k}\right)+2 H\left(r_{b}\right)\left(\sum_{k=1}^{n_{i}} w_{i j k}-M_{i}\right) .
$$

Equating equation (15) to zero and solving for $w_{i j k}$ the study obtained;

$$
w_{i j k}=\frac{z_{l j k}-H\left(r_{b}\right) q_{i j k} z_{l j k}\left(\sum_{\substack{k=1 \\ p \neq k}}^{n_{i}} w_{i j p}\left[\hat{h}_{i j k} \hat{h}_{i j p}+1\right]-\sum_{p=1}^{M_{i}}\left[\hat{h}_{i j k} \hat{h}_{i j p}-1\right]\right)}{\left.1+H\left(r_{b}\right)\left(\left(\hat{h}_{i j k}\right)^{2}+1\right) q_{i j k} z_{i j k}\right)} .
$$

Thus, a nonparametric estimator of the cluster total is given as;

$$
\hat{t}_{i}=\sum_{i \varepsilon c} w_{i j k} \hat{y}_{i j k}=\sum_{k=1}^{n_{i}} \frac{y_{i j k} z_{i j k}}{1+H\left(r_{b}\right)\left(\left(\left(\hat{h}_{i j k}\right)^{2}+1\right) q_{i j k} z_{j k l}\right)}-\sum_{k=1}^{n_{i}} \frac{H\left(r_{b}\right) q_{i j k} z_{l j k} y_{i j k}\left(\sum_{\substack{k=1 \\ p \neq k}}^{n_{i}} w_{i j p}\left[\hat{h}_{i j k} \hat{h}_{i j p}+1\right]-\sum_{p=1}^{M_{i}}\left[\hat{h}_{i j k} \hat{h}_{i j p}-1\right]\right)}{1+H\left(r_{b}\right)\left(\left(\left(\hat{h}_{i j k}\right)^{2}+1\right) q_{i j k} z_{i j k}\right)} .
$$

Secondly, the nonparametric fit of the cluster totals based on penalized splines in this study was defined as;

$$
\hat{h}_{t_{i}}=J_{p s i}^{T} \hat{t}_{c}
$$

where $J_{p s i}^{T}$ is as defined by Nthiwa Janiffer Mwende et al. (2020), [8] as

$$
J_{p s i}^{T}=X_{r i}\left(X_{r c}{ }^{T} W_{c} X_{r c}+A \alpha\right)^{-1} X_{r c}{ }^{T} W_{c}
$$

in which a matrix $X_{r}$ has the rows

$$
X_{r i}^{T}=\left\{1, x_{i}, \ldots, x_{i}^{q},\left(x_{i}-k_{1}\right)_{+}^{q}, \ldots,\left(x_{i}-k_{l}\right)_{+}^{q}\right\}
$$

for $i \varepsilon G$, further, $X_{r c}$ is the sub matrix of $X_{r}$ which consists of the rows $X_{r i}^{T}$ for which the cluster $i \varepsilon c$, while the diagonal matrix of inverse inclusion probabilities was defined the same way as Nthiwa Janiffer Mwende et al. (2020), [8] as; $W=\operatorname{diag}, i \in G\left\{\frac{1}{\pi_{i}}\right\}$ and its sample sub matrix defined as $W_{c}=\operatorname{diag}, i \in c\left\{\frac{1}{\pi_{i}}\right\}$.

The study then proposed a nonparametric penalized spline model calibrated population total estimator as;

$$
\hat{y}_{P S}=\sum_{i \varepsilon c} w_{i} \hat{t}_{i}
$$

The weight $w_{i}$ in this equation (20) was obtained by minimizing the chi square distance measure given as;

$$
\delta=\sum_{i \in c} \frac{\left(w_{i}-z_{i}\right)^{2}}{q_{i} z_{i}}
$$


subject to

$$
\left\{\begin{array}{l}
\sum_{i \varepsilon c} w_{i}=C \text { and } \\
\sum_{i=1}^{c} w_{i} \hat{h}_{t_{i}}=\sum_{i=1}^{C} \hat{h}_{t_{i}}
\end{array}\right.
$$

$$
\delta=\sum_{i \in c} \frac{\left(w_{i}-z_{i}\right)^{2}}{q_{i} z_{i}}
$$

subject to

where $z_{i}=\frac{1}{\pi_{i}}$ and $q_{i}$ are some known positive constants uncorrelated with $z_{i}$. This, therefore, gave an optimization problem of;

minimize

$$
\left\{\begin{array}{l}
S_{1}(w)=\sum_{i=1}^{c} w_{i}-C=0 \\
S_{2}(w)=\sum_{i=1}^{c} w_{i} \hat{h}_{t_{i}}-\sum_{i=1}^{C} \hat{h}_{t_{i}}=0
\end{array}\right.
$$

which gave a penalty function of the form;

$$
\tau\left(w, r_{b}, \hat{h}_{t_{i}}\right)=\sum_{i \in c} \frac{\left(w_{i}-z_{i}\right)^{2}}{q_{i} z_{i}}+H\left(r_{b}\right)\left(\sum_{i=1}^{c} w_{i} \hat{h}_{t_{i}}-\sum_{i=1}^{c} \hat{h}_{t_{i}}\right)^{2}+H\left(r_{b}\right)\left(\sum_{i=1}^{c} w_{i}-C\right)^{2} .
$$

Following same penalty procedure as in estimating the optimal weights $w_{i j k}$ by penalty method above, the weight $w_{i}$ becomes;

$$
w_{i}=\frac{z_{i}-H\left(r_{b}\right) q_{i} z_{l}\left(\sum_{\substack{i=1 \\ j \neq i}}^{c} w_{j}\left[\hat{h}_{t_{i}} \hat{h}_{t_{j}}+1\right]-\sum_{j=1}^{C}\left[\hat{h}_{t_{i}} \hat{h}_{t_{j}}-1\right]\right)}{1+H\left(r_{b}\right)\left(\left(\left(\hat{h}_{t_{i}}\right)^{2}+1\right) q_{i} z_{l}\right)} .
$$

The weighted nonparametric estimator of population total based on p-splines when information is available at both cluster and element level as defined in equation (20) was obtained as;

$$
\hat{y}_{P S B}=\sum_{i \varepsilon c} w_{i} \hat{t}_{i}=\sum_{i \in c} \frac{H\left(r_{b}\right) q_{i} z_{l} \hat{t}_{i}\left(\sum_{\substack{i=1 \\ j \neq i}}^{c} w_{j}\left[\hat{h}_{t_{i}} \hat{h}_{t_{j}}+1\right]-\sum_{j=1}^{c}\left[\hat{h}_{t_{i}} \hat{h}_{t_{j}}-1\right]\right)}{1+H\left(r_{b}\right)\left(\left(\left(\hat{h}_{t_{i}}\right)\left(\left(\hat{h}_{t_{i}}\right)^{2}+1\right) q_{i} z_{l}\right)\right.}-\sum_{i \in c} \frac{\left.q_{i} z_{l}\right)}{1+H}
$$

\section{Penalty Function Method of Obtaining the Weights}

To obtain the within cluster weights, $W_{i j k}=\left(k=1,2, \ldots, n_{i}\right)$ and cluster level weights $w_{i},(i=1,2, \ldots, c)$, this study applied iterative procedure. Firstly, to obtain the within cluster weights, $W_{i j k}=\left(k=1,2, \ldots, n_{i}\right)$ the study solved the penalty function in equation (14) as an unconstrained minimization problem. The research in this case started with some initial guess for $w_{i j k}$ and $r_{b}$ then iteratively improved on the initial values until optimal values are obtained. The present study, therefore, followed the Newton method of unconstrained optimization, according to [8] as follows;

If $W_{i}=\left(w_{i j 1}, w_{i j 2}, \ldots, w_{i j n_{i}}\right)$ is let to be the set of the weights, of interest was to obtain $W_{i}^{*}$ such that

$$
\Gamma\left(W_{i}^{*}\right)=\left[\tau^{\prime}\left(w_{i j 1}, r_{b}\right), \ldots, \tau^{\prime}\left(w_{i j n_{i}}, r_{b},\right)\right]^{\prime}=0 .
$$

Further if $W_{i u}$ is let to be initial estimate of $W_{i}^{*}$ so that $W_{i}^{*}=W_{i u}+T_{i}$. The Taylor's series expansion of $\Gamma\left(W_{i}^{*}\right)$ gives

$$
\Gamma\left(W_{i}^{*}\right)=\Gamma\left(W_{i u}+T_{i}\right)=\Gamma\left(W_{i}\right)+J_{W_{i u}} T_{i}+\ldots .
$$

By neglecting the higher-order terms in the above equation (28) and setting $\Gamma\left(W^{*}\right)=0$ the study had

$$
\Gamma\left(w_{\text {iu }}\right)+J_{w_{\text {iu }}} T_{i}=0
$$

where $J_{w_{i u}}$ is a $n_{i}$ by $n_{i}$ matrix of second derivatives of the penalty function equation (15) evaluated at $W_{i u}$. Let also $i$ and $j$ be the row and column counters respectively with $i=\left(1,2, \ldots, n_{i}\right)$ rows with $j=\left(1,2, \ldots, n_{i}\right)$ columns. The matrix 
$J_{w_{i} u}$ has elements

$\frac{2}{q_{i j k} z_{i j k}}+2 H\left(r_{b}\right)\left(\left(\hat{h}_{i j k}\right)^{2}+1\right)$ in the main diagonal and elements $2 H\left(r_{b}\right)\left(\hat{h}_{i j k} \hat{h}_{i j p}+1\right)$ elsewhere.

If $J_{w_{u i}}$ is a nonsingular matrix, then, from the set of linear equations (29) we have for vector $\mathrm{T}$

$$
T_{i}=J^{-1}{ }_{u i} \Gamma\left(w_{i u}\right) .
$$

The following iterative procedure is used to find the improved approximations of $W_{i}^{*}$

$$
W_{i+1}=W_{i}+T_{i}=W_{i}-J_{w_{i}}^{-1} \Gamma\left(W_{i}\right)
$$

The sequence of the points $W_{i j 1}, W_{i j 2}, \ldots, W_{i j(u+1)}$ eventually converges to the actual solution $W_{i}^{*}$. Now, if we let $W_{i b}{ }^{*}$ be the minimum of $W_{i}^{*}$ obtained for a particular penalty, $r_{b}$ the study obtained a sequence of minimum points $W_{i j 1}{ }^{*}, W_{i j 2}^{*}, \ldots, W_{i j(b+1)}^{*}$ for the penalties $r_{1}, r_{2} \ldots, r_{b+1}$ until $W_{i b}{ }^{*}=W_{i j(b+1)}^{*}$ or $\tau\left(w_{i j k}, r_{b}, \hat{h}_{i j k}\right)=\tau\left(w_{i j k}, r_{b+1}, \hat{h}_{i j k}\right)$ for some specified accuracy level. The accuracy level may for example be, to certain decimal points or significance level. In addition, the penalty values may be set such that the starting point $r_{1}>0$ and $r_{b+1}=s r_{b}$, where $s<1, H\left(r_{b}\right) \rightarrow \infty$ as $r_{b} \rightarrow 0$.

This study applied iterative procedure to obtain the cluster level weights $w_{i},(i=1,2, \ldots, c)$. The penalty function in equation (24) was solved as an unconstrained minimization problem in a similar manner as within cluster weights; $W_{i j k}$ discussed in this section.

\section{Empirical Analysis and Discussions}

In the simulation study, a population of size 10,000 $(200 * 50=10,000)$ was simulated from a population structure containing 200 clusters each of size 50 . Each cluster had 5 strata of size 10 each. At stage one 10, 20, 30,.., 190 clusters were sampled from the 200 clusters by simple random sampling while at stage two, 5 elements where drawn from each stratum by proportional allocation. This gave sample of 25 elements from each of the sampled clusters. 10 replications per each sample size were generated. For penalized spline method, the number of knots and the Spline penalty were optimally generated.

Using $\mathrm{R}$ program, a population of independent and identically distributed variable $x$ was simulated using uniform $(0,1)$. This study used penalized spline equation (18) to fit cluster totals and equation (7) to fit for elements within a cluster. Using the auxiliary variable $x$, five populations for the dependent random variable for cluster element, and cluster total; $t_{i}$ were generated with the five functions as:

Linear function (Lin); $t_{i}=3+6 x$

Quadratic function $(\mathrm{Qd}) ; t_{i}=(20+6 x)^{2}$

Exponential function $(\operatorname{Exp}) ; t_{i}=20-\exp (x / 40)$

Cycle 4 function $(\mathrm{C} 4) ; t_{i}=0.5-\sin (8 \pi x)$

Cycle 2 function $(\mathrm{C} 2) ; t_{i}=0.5-\sin (2 \pi x)$

where $t_{i}$ is the ith cluster total and $x_{i}$ is cluster auxiliary variable known at the cluster level. The $\mathrm{s} k^{\text {th }}$ unit in $j^{\text {th }}$ stratum of $i^{t h}$ cluster was given by;

$$
y_{i j k}=\frac{t_{i}}{\text { cluster size }}+\text { error term }\left(e_{i}\right) / \sqrt{\text { cluster size }} .
$$

This study differentiated the five strata from each other by the following errors;

$e_{1}=\operatorname{runif}(-0.001,+0.001)$ for stratum $1, e_{2}=$ runif $(-0.002,+0.002)$ for stratum 2 , $e_{3}=\operatorname{runif}(-0.003,+0.003)$ for stratum $3, e_{4}=$ runif $(-0.004,+0.004)$ for stratum 4 and $e_{5}=$ runif $(-0.005,+0.005)$ for stratum 5 .

On the other hand, the respective kth auxiliary variable in the jth stratum of ith cluster was given as;

$$
\begin{gathered}
x_{i j k}=\frac{\left(3-y_{i j k}\right)}{6}+e_{j} \text { for linear } \\
x_{i j k}=\frac{20-\sqrt{y_{i j k}}}{6}+e_{j} \text { for quadratic } \\
x_{i j k}=\left(\log \left(20-y_{i j k}\right)\right) 40+e_{j} \text { for exponential } \\
x_{i j k}=\frac{\sin ^{-1}\left(y_{i j k}-0.5\right)}{8}+e_{j} \text { for cycle } 4 \\
x_{i j k}=\frac{\sin ^{-1}\left(y_{i j k}-0.5\right)}{2}+e_{j} \text { for cycle } 2 .
\end{gathered}
$$

This study reports on the performance penalized spline model calibrated estimator and its efficiency in comparison with Horvitz Thompson estimator. The performance of the nonparametric estimator; $\hat{y}_{P S}$ was evaluated using its relative bias $R_{B}$ and relative efficiency $R_{E}$. The relative bias was defined as

$$
R_{B}=\frac{\sum_{r=1}^{R}\left(y_{P S}-Y_{A T}\right)}{R^{*} Y_{A T}}
$$

where, $Y_{A T}$ is the actual total and $\mathrm{R}$ is the replicate number of samples. The relative efficiency was defined as 


$$
R_{E}=\frac{\operatorname{MSE}\left(y_{P S}\right)}{\operatorname{MSE}\left(y_{H T}\right)} .
$$

Large values of relative efficiencies represent higher efficiency for the design estimator $y_{H T}$ over the estimator $\hat{y}_{P S}$ and vice versa. The $\hat{y}_{H T}$ estimator was defined as $\hat{y}_{H T}=\sum z_{i} t_{i}$ where $z_{i}$ is the inverse of the inclusion probability given by $z_{i}=\frac{\mathrm{C}}{\mathrm{c}}$ for class total and $z_{i j k}=\frac{\text { cluster size }}{\text { sample size }}$ for cluster element. The estimator $\hat{y}_{H T}$ was used as the baseline comparison.

\subsection{Normality Test}

A One-sample Kolmogorov-Smirnov test was carried out in this study to test for normality of the nonparametric estimators; $\hat{y}_{P S}$ and the design estimator; Horvitz Thompson estimator; $\hat{y}_{H T}$. The $\mathrm{p}$ values at $\alpha=0.05$ for the five population mean functions obtained are as in table 1 below. A $\mathrm{p}$-value greater than the set $\alpha=0.05$ significance level means normality is established. The results show that at $\alpha=0.05$ the proposed estimators are normal for all the five functions.

Table 1. Normality test.

\begin{tabular}{lcc}
\hline Estimator & $\hat{y}_{P S}$ & $\hat{y}_{H T}$ \\
\hline Linear & 0.9928 & 0.8869 \\
Quadratic & 0.3763 & 0.3934 \\
Exponential & 0.7678 & 0.6876 \\
Cycle 4 & 0.8850 & 0.5584 \\
Cycle 2 & 0.9920 & 0.4319 \\
\hline
\end{tabular}

\subsection{Results for Population Total Estimates}

The results in tables 2, 3, 4, 5 and 6 below shows the actual total and the estimates of the penalized splines and the Horvitz Thompson for the respective mean functions with sample sizes; 50, 100 and 150. From the results the estimator $\hat{y}_{P S}$ are seen to give estimates that are close the actual total and also to those of Horvitz Thompson design estimator for all the 5 population functions.

Table 2. Linear Population total estimates for samples of sizes, 20, 50, 100 and 150.

\begin{tabular}{|c|c|c|c|c|c|c|c|c|c|c|c|}
\hline \multicolumn{2}{|c|}{$\begin{array}{l}\text { Replication } \\
\text { Number }\end{array}$} & 1 & 2 & 3 & 4 & 5 & 6 & 7 & 8 & 9 & 10 \\
\hline \multirow[b]{2}{*}{$y_{A T}$} & Sample size & & & & & & & & & & \\
\hline & $50 / 100 / 150$ & 4543.206 & 4543.206 & 4543.206 & 4543.206 & 4543.206 & 4543.206 & 4543.206 & 4543.206 & 4543.206 & 4543.206 \\
\hline \multirow{3}{*}{$\hat{y}_{P S}$} & 50 & 4550.709 & 4508.628 & 4568.743 & 4598.951 & 4526.772 & 4580.126 & 4506.070 & 4555.463 & 4486.108 & 4551.931 \\
\hline & 100 & 4525.000 & 4568.034 & 4568.753 & 4515.870 & 4568.810 & 4531.189 & 4563.055 & 4615.942 & 4512.589 & 4568.554 \\
\hline & 150 & 4537.296 & 4537.509 & 4522.132 & 4561.317 & 4515.272 & 4546.944 & 4528.760 & 4560.889 & 4535.451 & 4521.857 \\
\hline \multirow{3}{*}{$\hat{y}_{H T}$} & 50 & 4543.147 & 4505.097 & 4559.542 & 4598.436 & 4531.062 & 4576.261 & 4503.652 & 4551.989 & 4488.678 & 4544.956 \\
\hline & 100 & 4522.916 & 4566.846 & 4561.118 & 4516.681 & 4561.496 & 4532.834 & 4568.969 & 4620.047 & 4520.402 & 4565.650 \\
\hline & 150 & 4536.349 & 4539.143 & 4518.782 & 4563.124 & 4516.328 & 4540.459 & 4526.952 & 4556.363 & 4532.178 & 4525.400 \\
\hline
\end{tabular}

Table 3. Quadratic Population total estimates for samples of sizes, 20, 50, 100 and 150.

\begin{tabular}{llllllllllll}
\hline $\begin{array}{l}\text { Replication } \\
\text { Number }\end{array}$ & $\mathbf{1}$ & $\mathbf{2}$ & $\mathbf{3}$ & $\mathbf{4}$ & $\mathbf{5}$ & $\mathbf{6}$ & $\mathbf{7}$ & $\mathbf{8}$ & $\mathbf{9}$ & $\mathbf{1 0}$ \\
\hline \multirow{2}{*}{$y_{A T}$} & Sample size & & & & & & & & & & \\
& $50 / 100 / 150$ & 58508.64 & 58508.64 & 58508.64 & 58508.64 & 58508.64 & 58508.64 & 58508.64 & 58508.64 & 58508.64 & 58508.64 \\
& 50 & 59832.17 & 58626.97 & 55916.10 & 59068.46 & 60239.68 & 61313.31 & 56243.05 & 56767.31 & 58457.40 & 55972.48 \\
$\hat{y}_{P S}$ & 100 & 59208.13 & 59121.51 & 58549.64 & 58667.37 & 58077.49 & 58327.13 & 58899.17 & 58961.40 & 59057.99 & 58351.96 \\
& 150 & 58930.17 & 58508.48 & 58202.29 & 58577.28 & 58844.61 & 58348.84 & 58376.42 & 58062.99 & 57828.53 & 58361.61 \\
& 50 & 59835.06 & 58622.55 & 55912.85 & 59073.66 & 60238.51 & 61314.53 & 56252.56 & 56768.40 & 58452.97 & 55964.32 \\
$\hat{y}_{H T}$ & 100 & 59208.76 & 59120.87 & 58549.63 & 58665.68 & 58075.85 & 58331.41 & 58893.50 & 58961.65 & 59061.60 & 58348.58 \\
& 150 & 58932.61 & 58505.37 & 58205.57 & 58576.88 & 58851.44 & 58347.17 & 58379.05 & 58063.35 & 57824.62 & 58364.26 \\
\hline
\end{tabular}

Table 4. Exponential Population total estimates for samples of sizes, 20, 50, 100 and 150.

\begin{tabular}{llllllllllll}
\hline $\begin{array}{l}\text { Replication } \\
\text { Number }\end{array}$ & $\mathbf{1}$ & $\mathbf{2}$ & $\mathbf{3}$ & $\mathbf{4}$ & $\mathbf{5}$ & $\mathbf{6}$ & $\mathbf{7}$ & $\mathbf{8}$ & $\mathbf{9}$ & $\mathbf{1 0}$ \\
\hline \multirow{2}{*}{$y_{A T}$} & Sample size & & & & & & & & & \\
& $50 / 100 / 150$ & 3794.735 & 3794.735 & 3794.735 & 3794.735 & 3794.735 & 3794.735 & 3794.735 & 3794.735 & 3794.735 & 3794.735 \\
& 50 & 3799.913 & 3800.427 & 3800.963 & 3792.627 & 3798.025 & 3795.023 & 3787.908 & 3790.535 & 3807.945 & 3783.372 \\
$\hat{y}_{P S}$ & 100 & 3790.318 & 3794.567 & 3790.789 & 3791.142 & 3794.270 & 3795.132 & 3793.077 & 3795.497 & 3797.233 & 3794.940 \\
& 150 & 3794.733 & 3793.256 & 3790.879 & 3795.593 & 3790.908 & 3796.495 & 3799.995 & 3792.718 & 3794.792 & 3795.983 \\
& 50 & 3800.172 & 3800.242 & 3800.665 & 3792.678 & 3797.854 & 3795.050 & 3787.943 & 3790.483 & 3807.787 & 3783.129 \\
$\hat{y}_{H T}$ & 100 & 3790.028 & 3794.767 & 3790.476 & 3791.191 & 3794.124 & 3795.143 & 3793.297 & 3795.539 & 3797.097 & 3794.861 \\
& 150 & 3794.631 & 3793.194 & 3790.718 & 3795.681 & 3790.801 & 3796.415 & 3800.001 & 3792.819 & 3794.634 & 3795.881 \\
\hline
\end{tabular}


Table 5. Cycle 4 population total estimates for samples of sizes, 20, 50, 100 and 150.

\begin{tabular}{lllllll}
\hline \multicolumn{2}{l}{ Replication Number } & $\mathbf{1}$ & $\mathbf{2}$ & $\mathbf{3}$ & $\mathbf{4}$ & $\mathbf{5}$ \\
\hline \multirow{2}{*}{$y_{A T}$} & Sample size & & & & & \\
& $50 / 100 / 150$ & 98.12195 & 98.12195 & 98.12195 & 98.12195 & 98.12195 \\
$\hat{y}_{P S}$ & 50 & 106.74650 & 132.50628 & 119.94744 & 89.20880 & 85.34613 \\
& 100 & 96.41683 & 90.02801 & 97.57377 & 100.66343 & 94.51954 \\
& 150 & 99.74723 & 90.42254 & 93.29842 & 102.81139 & 104.91942 \\
$\hat{y}_{H T}$ & 50 & 113.48154 & 131.49654 & 125.62301 & 95.99730 & 79.22657 \\
& 100 & 94.70033 & 92.00542 & 99.25881 & 102.30048 & 94.31615 \\
\\
\hline
\end{tabular}

Table 5. Continued.

\begin{tabular}{lllllll}
\hline \multicolumn{2}{l}{ Replication Number } & $\mathbf{6}$ & $\mathbf{7}$ & $\mathbf{8}$ & $\mathbf{9}$ & $\mathbf{1 0}$ \\
\hline$y_{A T}$ & Sample size & & & & & \\
& $50 / 100 / 150$ & 98.12195 & 98.12195 & 98.12195 & 98.12195 & 98.12195 \\
$\hat{y}_{P S}$ & 50 & 95.97287 & 69.00133 & 137.85757 & 91.38317 & 94.34510 \\
& 100 & 109.77530 & 85.37745 & 96.69058 & 104.97689 & 93.86149 \\
& 150 & 97.10018 & 98.32416 & 87.04543 & 92.83903 & 98.93072 \\
$\hat{y}_{H T}$ & 50 & 92.69908 & 68.07357 & 142.53958 & 92.80498 & 88.05275 \\
& 100 & 110.22664 & 86.45460 & 95.15238 & 102.69284 & 93.62301 \\
& 150 & 99.12175 & 100.96804 & 86.27175 & 95.20490 & 100.40792 \\
\hline
\end{tabular}

Table 6. Cycle 2 population total estimates for samples of sizes, 20, 50, 100 and 150.

\begin{tabular}{llllllllllll}
\hline $\begin{array}{l}\text { Replication } \\
\text { Number }\end{array}$ & $\mathbf{1}$ & $\mathbf{2}$ & $\mathbf{3}$ & $\mathbf{4}$ & $\mathbf{5}$ & $\mathbf{6}$ & $\mathbf{7}$ & $\mathbf{8}$ & $\mathbf{9}$ & $\mathbf{1 0}$ \\
\hline \multirow{2}{*}{$y_{A T}$} & Sample size & & & & & & & & & & \\
& $50 / 100 / 150$ & 116.4859 & 116.4859 & 116.4859 & 116.4859 & 116.4859 & 116.4859 & 116.4859 & 116.4859 & 116.4859 & 116.4859 \\
& 50 & 92.01059 & 104.7750 & 113.7534 & 99.91309 & 84.01602 & 129.1396 & 141.9112 & 132.7674 & 123.5790 & 154.1871 \\
$\hat{y}_{P S}$ & 100 & 114.2216 & 114.2180 & 107.6319 & 121.8142 & 131.3348 & 121.3722 & 115.1419 & 100.68917 & 122.5620 & 112.1085 \\
& 150 & 114.7727 & 118.6039 & 120.1997 & 110.1363 & 112.3717 & 123.1632 & 122.7303 & 111.1371 & 120.6737 & 111.0792 \\
& 50 & 92.26469 & 104.9711 & 110.4062 & 100.62389 & 85.96008 & 138.7731 & 142.7816 & 116.2985 & 119.2736 & 148.2210 \\
$\hat{y}_{H T}$ & 100 & 109.9282 & 118.0110 & 111.7657 & 117.6613 & 129.6345 & 118.8748 & 116.8948 & 99.04232 & 114.9889 & 109.5154 \\
& 150 & 115.2668 & 116.3559 & 114.0293 & 110.9012 & 114.7852 & 123.9057 & 124.0244 & 112.8755 & 121.4621 & 110.0413 \\
\hline
\end{tabular}

\subsection{Results of Variances and Variance Ratios for Various Sample Size}

This section presents both the variance and variance ratio of the two estimators; $\hat{y}_{P S}$ based on a penalized spline and $\hat{y}_{H T}$ based on Horvitz Thompson and their respective graphs. The variance and variance ratios for different functions are summarized in table 7 , and their comparative graphs in figures 1, 2, 3, 4 and 5 for the respective functions.

\subsubsection{Tabular Results of Variances and Variance Ratios for Various Sample Size}

The table 7 below shows both variance and variance ratios of the two estimators based on penalized splines and Horvitz. The variances of the two estimators for the 5 functions decrease as the sample size increases implying that the estimators are consistent. From the table, the $\operatorname{var}\left(\hat{y}_{H T}\right)$ for linear and exponential functions are smaller than those of $\operatorname{var}\left(\hat{y}_{P S}\right)$ except for the 9 sample sizes; 20, 60, 70, 90, 110,
120, 130, 140 and 160 and 8 sample sizes 40, 70, 90, 100, 110, 130, 150 and 180 respectively. This comparison is evident from the variance ratio; $\operatorname{var}\left(\hat{y}_{P S} / \hat{y}_{H T}\right)$ for the same functions. For quadratic function the $\operatorname{var}\left(\hat{y}_{P S}\right)$ estimator is less variant than Horvitz Thompson estimator since $\operatorname{var}\left(\hat{y}_{P S}\right)$ is consistently lower than that of Thompson estimator for all samples sizes except for 5 sample sizes; 30, 70, 140, 170 and 180 and this applies to the variance ratio; $\operatorname{var}\left(\hat{y}_{P S} / \hat{y}_{H T}\right)$ for the same function. For cycle 4 and cycle 2 the $\operatorname{var}\left(\hat{y}_{H T}\right)$ is slightly less variant than that of $\operatorname{var}\left(\hat{y}_{P S}\right)$ in some samples except for the 7 sample sizes; 50, 60, 110, 120, 140, 160 and 180 and the 6 sample sizes; 20, 40, 60, 130, 140 and 170 for cycle 2 and all these is evident from the respective ratios; $\operatorname{var}\left(\hat{y}_{P S} / \hat{y}_{H T}\right)$. 
Table 7. Results of Variances and variance ratios for various sample size.

\begin{tabular}{|c|c|c|c|c|c|c|}
\hline Sample size & & 10 & 20 & 30 & 40 & 50 \\
\hline \multirow{5}{*}{$\operatorname{var}\left(\hat{y}_{P S}\right)$} & Lin & 3651.667479 & 3951.1194439 & 3543.5359584 & 3337.6837219 & 1278.906399 \\
\hline & Qd & $5,331,024$ & $3,522,390$ & $4,482,455$ & $972,450.3$ & $3,728,991$ \\
\hline & Exp & 168.278885 & 169.0653534 & 51.0707619 & 47.3712646 & 52.4873479 \\
\hline & $\mathrm{C} 4$ & 2904.529547 & 978.5458207 & 505.7774565 & 557.0358257 & 477.5578929 \\
\hline & $\mathrm{C} 2$ & 835.9659326 & 1292.6891074 & 190.0423932 & 221.8191635 & 511.4902605 \\
\hline \multirow{5}{*}{$\operatorname{var}\left(\hat{y}_{H T}\right)$} & Lin & 3441.982023 & 4170.5863917 & 3293.8879518 & 3320.0194090 & 1171.349553 \\
\hline & Qd & $5,351,925$ & $3,529,463$ & $4,477,180$ & $973,958.5$ & $3,731,934$ \\
\hline & Exp & 163.048127 & 163.9132225 & 50.3517091 & 48.5950529 & 52.3219643 \\
\hline & $\mathrm{C} 4$ & 2609.500036 & 960.9689888 & 382.5380028 & 546.3226956 & 584.9590572 \\
\hline & $\mathrm{C} 2$ & 747.9882971 & 1358.9711656 & 169.4059765 & 231.5295217 & 459.9957258 \\
\hline \multirow{5}{*}{$\operatorname{var}\left(\hat{y}_{P S}\right) / \operatorname{var}\left(\hat{y}_{H T}\right)$} & Lin & 1.060920 & 0.9473774 & 1.0757913 & 1.0053205 & 1.091823 \\
\hline & Qd & 0.9960946 & 0.9979960 & 1.001178 & 0.9984514 & 0.9 .992114 \\
\hline & Exp & 1.032081 & 1.0314321 & 1.0142806 & 0.9748166 & 1.0031609 \\
\hline & $\mathrm{C} 4$ & 1.113060 & 1.0182907 & 1.3221626 & 1.0196095 & 0.8163954 \\
\hline & $\mathrm{C} 2$ & 1.1176190 & 0.9512263 & 1.1218163 & 0.9580600 & 1.1119457 \\
\hline Sample size & & 60 & 70 & 80 & 90 & 100 \\
\hline & Lin & 1686.3531718 & 683.4909103 & 739.351333 & 621.8465705 & 1032.696102 \\
\hline & Qd & $1,432,228$ & $795,246.2$ & $1,100,148$ & $239,576.3$ & $148,716.9$ \\
\hline $\operatorname{var}\left(\hat{y}_{P S}\right)$ & Exp & 37.5194780 & 10.8705338 & 12.2096085 & 17.0080090 & 5.2460144 \\
\hline & $\mathrm{C} 4$ & 221.6688694 & 288.1115958 & 114.015931 & 84.9656554 & 48.9687456 \\
\hline & $\mathrm{C} 2$ & 235.0572714 & 178.749618 & 364.9281303 & 334.5377284 & 74.1583869 \\
\hline & $\operatorname{lin}$ & 1798.2234675 & 721.2150960 & 730.283623 & 640.0131115 & 992.073248 \\
\hline & Qd & 1,434661 & $793,026.8$ & $1,101,905$ & $239,976.3$ & $148,954.9$ \\
\hline $\operatorname{var}\left(\hat{y}_{H T}\right)$ & Exp & 36.9130210 & 11.5590297 & 12.1355654 & 17.2458368 & 5.5536149 \\
\hline & $\mathrm{C} 4$ & 222.6924910 & 285.6353110 & 107.396674 & 79.9022833 & 44.8840996 \\
\hline & $\mathrm{C} 2$ & 265.1027829 & 147.086222 & 338.3337506 & 332.5792995 & 63.1641415 \\
\hline & Lin & 0.9377884 & 0.9476936 & 1.012417 & 0.9716154 & 1.040947 \\
\hline & Qd & 0.9983042 & 1.002799 & 0.9984059 & 0.9983334 & 0.9984022 \\
\hline $\operatorname{var}\left(y_{P S}\right) / \operatorname{var}\left(\hat{\hat{y}}_{U T}\right)$ & Exp & 1.0164293 & 0.9404365 & 1.0061013 & 0.9862096 & 0.9446126 \\
\hline$/ \operatorname{var}\left(y_{H T}\right)$ & $\mathrm{C} 4$ & 0.9954034 & 1.0086694 & 1.061634 & 1.0633696 & 1.0910043 \\
\hline & $\mathrm{C} 2$ & 0.8866647 & 1.215271 & 1.0786040 & 1.0058886 & 1.1740583 \\
\hline Sample size & & 110 & 120 & 130 & 140 & 150 \\
\hline & Lin & 528.8463282 & 391.1319758 & 367.6803949 & 366.0277604 & 250.3687311 \\
\hline & Qd & $552,643.4$ & $566,478.0$ & $397,977.0$ & $205,447.0$ & $111,742.5$ \\
\hline $\operatorname{var}\left(\hat{y}_{P S}\right)$ & Exp & 4.0804940 & 12.6728286 & 8.8227916 & 4.0807057 & 7.6035401 \\
\hline & $\mathrm{C} 4$ & 53.0041054 & 62.3777231 & 49.178826 & 45.8120890 & 31.2021121 \\
\hline & $\mathrm{C} 2$ & 51.9719975 & 102.0590988 & 39.9900411 & 78.6794029 & 26.379535 \\
\hline & Lin & 563.9793390 & 418.7877861 & 414.6097232 & 377.8959278 & 230.0089091 \\
\hline & Qd & $555,015.1$ & $569,733.0$ & $398,328.6$ & $205,206.4$ & 112,9230 \\
\hline $\operatorname{var}\left(\hat{y}_{H T}\right)$ & Exp & 4.3935972 & 12.3957597 & 8.97661099 & 3.820459 & 7.7526387 \\
\hline & $\mathrm{C} 4$ & 73.9513379 & 65.9388513 & 35.895394 & 51.8420740 & 27.0439387 \\
\hline & $\mathrm{C} 2$ & 43.2446770 & 89.7462109 & 41.2334476 & 82.1997916 & 25.854080 \\
\hline & Lin & 0.9377051 & 0.9339622 & 0.8868108 & 0.9685941 & 1.0885175 \\
\hline & Qd & 0.9957268 & 0.9942867 & 0.9991173 & 1.001173 & 0.9895455 \\
\hline $\operatorname{var}\left(\hat{y}_{P S}\right) / \operatorname{var}\left(\hat{y}_{H T}\right)$ & Exp & 0.9287365 & 1.0223519 & 0.9828644 & 1.0681190 & 0.9807680 \\
\hline & $\mathrm{C} 4$ & 0.7167430 & 0.9459935 & 1.370060 & 0.8836855 & 1.1537562 \\
\hline & $\mathrm{C} 2$ & 1.2018126 & 1.1371967 & 0.9698447 & 0.9571728 & 1.020324 \\
\hline Sample size & & 160 & 170 & 180 & & 190 \\
\hline & Lin & 157.6309214 & 179.052477 & 65.0 & & 28.0041116 \\
\hline & Qd & $253,692.2$ & $47,602.45$ & 76,4 & & $32,023.10$ \\
\hline $\operatorname{var}\left(\hat{y}_{P S}\right)$ & Exp & 3.657975 & 3.4205472 & 2.31 & & 1.922969 \\
\hline & $\mathrm{C} 4$ & 28.7065251 & 11.8576488 & 17.4 & & 7.9386513 \\
\hline & $\mathrm{C} 2$ & 25.326064 & 23.6213578 & 11.0 & & 7.680566 \\
\hline & Lin & 168.0263317 & 136.965407 & 61.7 & & 21.0822213 \\
\hline & Qd & $254,282.6$ & $47,307.85$ & 76,2 & & 32262.74 \\
\hline $\operatorname{var}\left(\hat{y}_{H T}\right)$ & Exp & 3.602958 & 3.3717028 & 2.46 & & 1.787780 \\
\hline & $\mathrm{C} 4$ & 31.0215984 & 8.1252690 & 18.8 & & 4.2808000 \\
\hline & $\mathrm{C} 2$ & 14.801159 & 27.5178092 & 5.45 & & 2.981572 \\
\hline
\end{tabular}




\begin{tabular}{clllll}
\hline Sample size & & $\mathbf{1 6 0}$ & $\mathbf{1 7 0}$ & $\mathbf{1 8 0}$ & 1.0534708 \\
\hline & Lin & 0.9381323 & 1.307282 & 1.3283283 & 1.002053 \\
$\operatorname{var}\left(\hat{y}_{P S}\right) / \operatorname{var}\left(\hat{y}_{H T}\right)$ & Qd & 0.9976779 & 1.006227 & 0.9925723 & 1.9377138 \\
& Exp & 1.015270 & 1.0144866 & 0.9216670 & 1.8544784 \\
& C4 & 0.9253722 & 1.4593546 & 2.019298 \\
\hline
\end{tabular}

\subsubsection{Graphical Results of Variances and Variance Ratio for Various Sample Size}

Results in figures 1, 2, 3, 4, and 5 represent respectively, the graphical variance ratio for the five population functions; linear, quadratic, exponential, Cycle 4 and Cycle 2 respectively for the two estimators based on penalized splines and Horvitz Thompson. Results from the five figures show that the ratio $\operatorname{var}\left(\hat{y}_{P S} / \hat{y}_{H T}\right)$ was concentrated below one for quadratic function, implying that Thompson estimator is more variant than $\operatorname{var}\left(\hat{y}_{P S}\right)$ in quadratic. On the other side Horvitz Thompson estimator is less variant than $\operatorname{var}\left(\hat{y}_{P S}\right)$ in linear, exponential cycle 4 and cycle 2 functions because their ratios concentrate slightly above one.

\subsection{Relative Bias}

The following table 8 shows the values of relative biases for the five population functions. The results from the table show that the relative biases for the two estimates are minimal, given that the population totals were in thousands, and this point to unbiasedness. It is also noted that the difference of the relative biases of the penalized spline estimator with its corresponding Horvitz Thompson estimators for all the five population functions is not very significant. Hence, the proposed model calibrated estimator is unbiased.

Spline vs Horvitz Thompson Variance Ratio

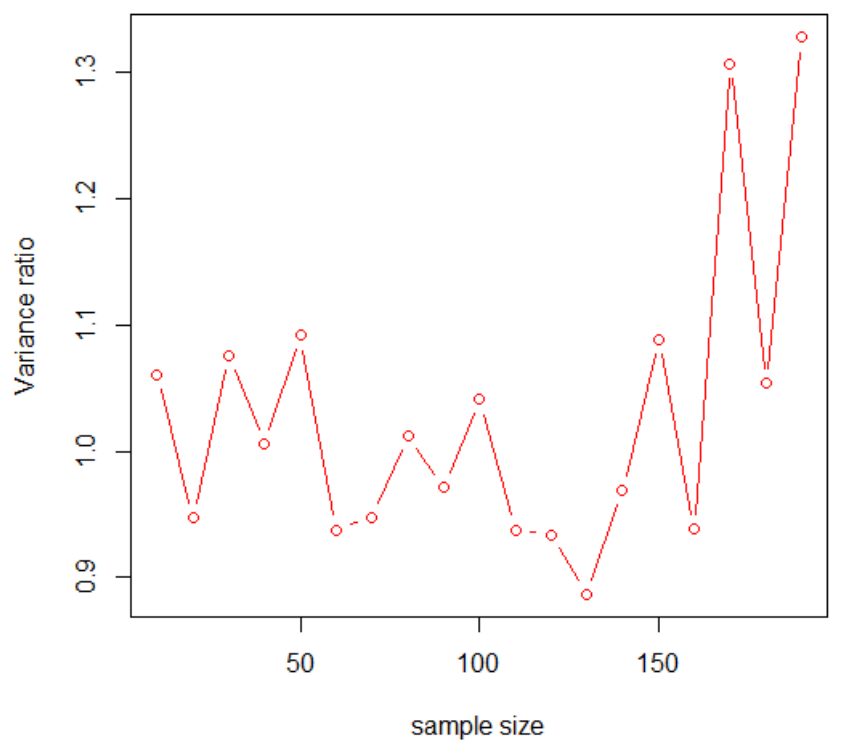

Figure 1. Linear.
Spline vs Horvitz Thompson Variance Ratio

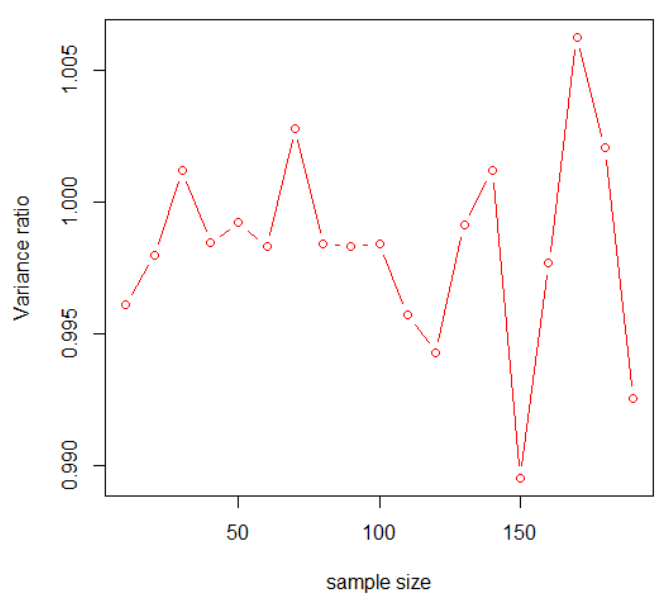

Figure 2. Quadratic.

Spline vs Horvitz Thompson Variance Ratio

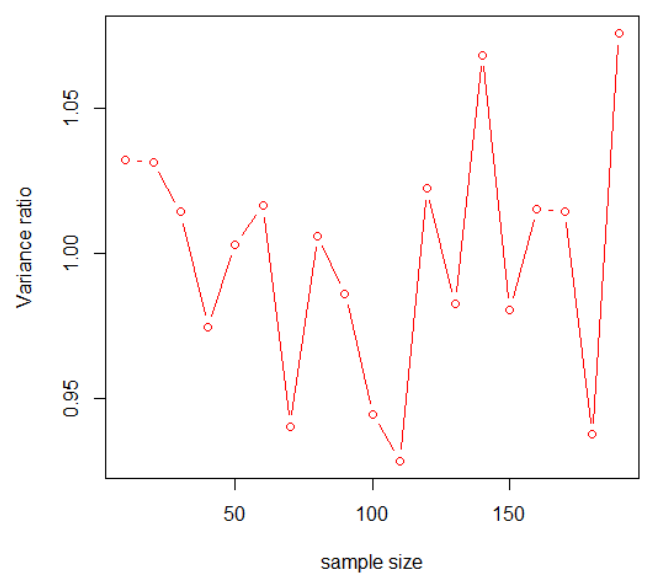

Figure 3. Exponential.

Spline vs Horvitz Thompson Variance Ratio

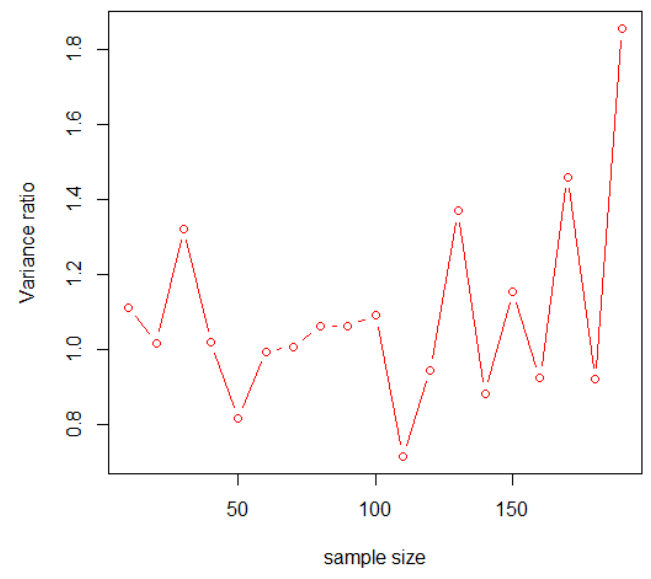

Figure 4. Cycle 4. 
Spline vs Horvitz Thompson Variance Ratio

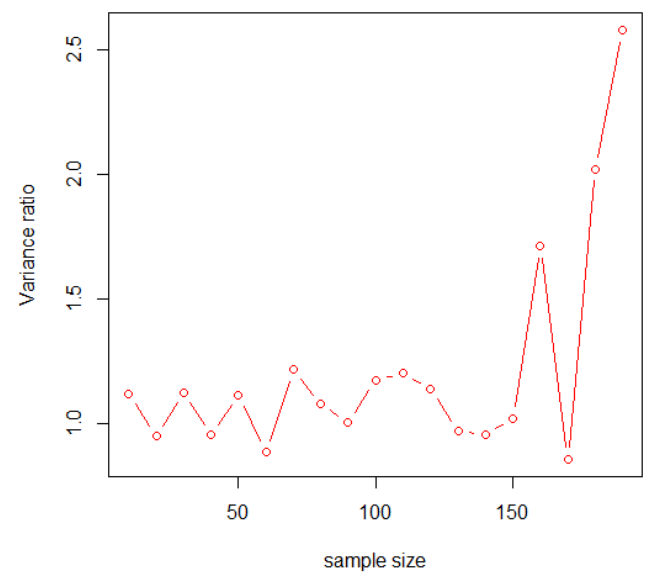

Figure 5. Cycle 2.

Table 8. Relative Biases for three estimators.

\begin{tabular}{lll}
\hline Estimator & $\hat{y}_{P S}$ & $\hat{y}_{H T}$ \\
\hline Linear & -0.0006773947 & -0.0006504365 \\
Quadratic & 0.002472271 & 0.002474501 \\
Exponential & 0.00005334967 & 0.00005276563 \\
Cycle 4 & -0.0004616429 & -0.0007526457 \\
Cycle 2 & 0.01028577 & 0.00724342 \\
\hline
\end{tabular}

4.5. Results on Relative Efficiency for Various Sample Sizes

\subsubsection{Tabular Results for MSE and MSE Ratios for Various Sample Size}

The MSE and relative efficiency (MSE Ratios) for the five different functions are summarized in table 9. Generally, the estimator with a smaller MSE is regarded as the most efficient one. From table 9 MSEs of $\hat{y}_{P S}$ is smaller than that of $\hat{y}_{H T}$ in some samples sizes but from other sample sizes the $\hat{y}_{H T}$ estimator has reduced MSE than the estimator; $\hat{y}_{P S}$. The relative efficiencies (MSE Ratios) in table 9 examines the robustness of the various population functions. There doesn't appear to be a clear noticeable performance difference of $\hat{y}_{P S}$ estimator in comparison to $\hat{y}_{H T}$ estimator. In some instances $\hat{y}_{P S}$ has a smaller error margin than $\hat{y}_{H T}$, while in other samples, $\hat{y}_{H T}$ has smaller error margins than the nonparametric model calibrated estimator; $\hat{y}_{P S}$. The $\hat{y}_{P S}$ estimator is more efficient than $\hat{y}_{H T}$ estimator in all the samples except for 5 sample sizes; 30, 70, 140, 170 and 180 for quadratic and 4 sample sizes; 30, 40, 110 and 140 for cycle 4 . In addition, $\hat{y}_{P S}$ estimator is slightly more efficient than $\hat{y}_{H T}$ estimator for exponential functions and cycle 2, except for 7 sample sizes; 10, 20, 70, 80, 120, 130 and 180 and 8 sample sizes; 30, 50, 60, 80, 90110,120 and 150 respectively. On the other hand the estimator; $\hat{y}_{H T}$ is slightly more efficient than $\hat{y}_{P S}$ estimator in all the samples except for the 9 sample sizes; 20, 40,60, 110, 120, 130, 150, 180 and 190 for linear function.

Table 9. Results of MSE and MSE ratios for various sample size.

\begin{tabular}{|c|c|c|c|c|c|c|}
\hline \multicolumn{2}{|c|}{ Sample size } & 10 & 20 & 30 & 40 & 50 \\
\hline \multirow{5}{*}{$\begin{array}{c}\text { MSE } \\
\hat{y}_{P S}\end{array}$} & Lin & 3761.901218 & 3997.1178405 & 3599.576631 & 3340.0779186 & 1278.927001 \\
\hline & Qd & $7,446,041$ & $4,738,647$ & $4,550,979$ & $984,679.2$ & $3,799,186$ \\
\hline & Exp & 170.6475187 & 169.410735 & 52.5931846 & 48.8811167 & 53.3688749 \\
\hline & $\mathrm{C} 4$ & 2943.9179205 & 1007.5783450 & 568.007902 & 583.4024883 & 494.4464803 \\
\hline & $\mathrm{C} 2$ & 1195.9670865 & 1530.1841661 & 271.0469163 & 227.3060849 & 512.7431068 \\
\hline \multirow{5}{*}{$\begin{array}{l}\text { MSE } \\
\hat{y}_{H T}\end{array}$} & Lin & 3529.915102 & 4219.3669187 & 3345.352957 & 3325.2848170 & 1179.902056 \\
\hline & Qd & $7,485,276$ & $4,744,751$ & $4,547,491$ & $986,394.6$ & $3,802,210$ \\
\hline & Exp & 167.6882442 & 159.122957 & 57.2071173 & 51.7141427 & 56.1322306 \\
\hline & $\mathrm{C} 4$ & 2903.0705831 & 1142.1461835 & 445.271374 & 468.4352616 & 641.6641625 \\
\hline & $\mathrm{C} 2$ & 1306.1443127 & 1686.9108741 & 202.1827495 & 296.3214559 & 414.9023874 \\
\hline \multirow{5}{*}{$\begin{array}{c}\text { MSE } \\
\hat{y}_{P S} \\
/ \mathrm{MSE} \\
\hat{y}_{H T}\end{array}$} & Lin & 1.010684 & 0.9841703 & 1.000111 & 0.9907866 & 1.024582 \\
\hline & Qd & 0.9947583 & 0.9987136 & 1.000767 & 0.9982609 & 0.9992047 \\
\hline & Exp & 1.0176475 & 1.064653 & 0.9193469 & 0.9452176 & 0.9507706 \\
\hline & $\mathrm{C} 4$ & 0.9440421 & 0.8657855 & 1.015070 & 1.1705200 & 0.9487043 \\
\hline & $\mathrm{C} 2$ & 0.9441625 & 0.9612389 & 1.0125236 & 0.8432357 & 1.1093576 \\
\hline \multicolumn{2}{|c|}{ Sample size } & 60 & 70 & 80 & 90 & 100 \\
\hline \multirow{5}{*}{$\begin{array}{c}\text { MSE } \\
\hat{y}_{P S}\end{array}$} & Lin & 2213.3671469 & 868.6904893 & 941.9650729 & 625.4252385 & 1144.486644 \\
\hline & Qd & $1,444,626$ & $824,239.5$ & $1,100,902$ & $239,875.2$ & $194,317.5$ \\
\hline & Exp & 38.555204 & 11.8924768 & 12.425321 & 17.0663297 & 6.3248485 \\
\hline & $\mathrm{C} 4$ & 241.8879912 & 288.1286386 & 115.2612613 & 101.4898390 & 50.2538319 \\
\hline & $\mathrm{C} 2$ & 285.2328794 & 199.2620918 & 396.7704457 & 335.674333 & 74.3001555 \\
\hline \multirow{5}{*}{$\begin{array}{l}\text { MSE } \\
\hat{y}_{H T}\end{array}$} & Lin & 2293.3339425 & 922.9854323 & 981.6586883 & 640.0297615 & 1102.101586 \\
\hline & Qd & $1,447,216$ & $822,176.1$ & $1,102,720$ & $240,235.0$ & $194,374.0$ \\
\hline & Exp & 42.740574 & 11.8107904 & 11.150557 & 17.9337831 & 6.9595295 \\
\hline & $\mathrm{C} 4$ & 346.3966814 & 309.8198703 & 108.1009965 & 115.8467571 & 55.8920469 \\
\hline & $\mathrm{C} 2$ & 337.3473346 & 188.3122440 & 356.2083152 & 273.639732 & 76.4726920 \\
\hline \multirow{5}{*}{$\begin{array}{l}\mathrm{MSE} \\
\hat{y}_{P S} \\
/ \mathrm{MSE} \\
\hat{y}_{H T}\end{array}$} & Lin & 0.9875441 & 1.0345659 & 1.0248652 & 1.0786348 & 1.003822 \\
\hline & Qd & 0.9982109 & 1.002510 & 0.9983508 & 0.9985023 & 0.9997094 \\
\hline & Exp & 0.902075 & 1.0069163 & 1.114323 & 0.9516302 & 0.9088040 \\
\hline & $\mathrm{C} 4$ & 0.7290980 & 0.9229237 & 0.9957634 & 0.7655590 & 0.8227334 \\
\hline & $\mathrm{C} 2$ & 1.0160905 & 0.8744224 & 1.0139206 & 1.217429 & 0.8709300 \\
\hline
\end{tabular}




\begin{tabular}{|c|c|c|c|c|c|c|}
\hline \multirow{5}{*}{ MSE $\hat{y}_{P S}$} & Lin & 655.8482220 & 477.6281396 & 367.6808541 & 379.2494284 & 292.1500391 \\
\hline & Qd & $607,022.7$ & $593,307.8$ & $415,432.1$ & $267,156.0$ & $122,666.1$ \\
\hline & Exp & 4.1202120 & 13.494986 & 9.7373345 & 6.7647846 & 7.6434632 \\
\hline & $\mathrm{C} 4$ & 83.495772 & 77.3385387 & 50.1603724 & 50.3699637 & 33.6924933 \\
\hline & $\mathrm{C} 2$ & 52.271479 & 104.7423976 & 40.0147599 & 79.4145996 & 26.3795354 \\
\hline \multirow{5}{*}{ MSE $\hat{y}_{H T}$} & Lin & 700.5660838 & 545.2801802 & 415.5757672 & 382.8570384 & 289.2821260 \\
\hline & Qd & $608,585.2$ & $596,561.5$ & $415,988.3$ & $267,093.0$ & $123,657.2$ \\
\hline & Exp & 4.4396357 & 13.214463 & 9.8181521 & 6.5505387 & 7.8190212 \\
\hline & $\mathrm{C} 4$ & 104.381583 & 91.3097778 & 39.0132544 & 51.7093668 & 40.4558555 \\
\hline & $\mathrm{C} 2$ & 39.431819 & 89.9134820 & 41.9892032 & 90.0818898 & 22.8535505 \\
\hline \multirow{5}{*}{$\begin{array}{l}\text { MSE } \hat{y}_{P S} / \mathrm{MSE} \\
\hat{y}_{H T}\end{array}$} & Lin & 0.9538936 & 0.9867949 & 0.9313259 & 1.0079811 & 0.9156409 \\
\hline & Qd & 0.9974326 & 0.9945459 & 0.9986628 & 1.000236 & 0.9919849 \\
\hline & Exp & 0.9107537 & 1.057114 & 1.0250755 & 0.9591387 & 0.9498795 \\
\hline & $\mathrm{C} 4$ & 1.106025 & 0.8855587 & 0.9287332 & 1.0412362 & 0.7055897 \\
\hline & $\mathrm{C} 2$ & 1.102070 & 1.0183366 & 0.9902773 & 0.9336432 & 1.1319365 \\
\hline \multicolumn{2}{|l|}{ Sample size } & 160 & 170 & \multicolumn{2}{|c|}{180} & 190 \\
\hline \multirow{5}{*}{ MSE $\hat{y}_{P S}$} & Lin & 165.6221865 & 179.918580 & \multicolumn{2}{|c|}{65.9200680} & 31.1640058 \\
\hline & Qd & $253,768.2$ & $62,777.18$ & \multicolumn{2}{|c|}{$76,511.68$} & $36,341.06$ \\
\hline & Exp & 3.9267096 & 3.4288437 & \multicolumn{2}{|c|}{2.8152284} & 3.7338132 \\
\hline & $\mathrm{C} 4$ & 47.4667990 & 15.1352895 & \multicolumn{2}{|c|}{23.7547279} & 8.0433500 \\
\hline & $\mathrm{C} 2$ & 32.9937376 & 23.6264682 & \multicolumn{2}{|c|}{14.1782831} & 9.0175933 \\
\hline \multirow{5}{*}{ MSE $\hat{y}_{H T}$} & Lin & 179.0923562 & 137.646389 & \multicolumn{2}{|c|}{65.1613352} & 24.2407817 \\
\hline & Qd & $254,336.6$ & $62,561.61$ & \multicolumn{2}{|c|}{$76,335.32$} & $36,612.13$ \\
\hline & Exp & 3.8768434 & 3.3762876 & \multicolumn{2}{|c|}{3.0369193} & 3.7901165 \\
\hline & $\mathrm{C} 4$ & 47.4120481 & 14.0677071 & \multicolumn{2}{|c|}{31.5174763} & 13.4859242 \\
\hline & $\mathrm{C} 2$ & 20.8959407 & 28.3225165 & \multicolumn{2}{|c|}{8.3965927} & 7.2576928 \\
\hline \multirow{5}{*}{$\begin{array}{l}\text { MSE } \hat{y}_{P S} / \mathrm{MSE} \\
\hat{y}_{H T}\end{array}$} & Lin & 1.0137243 & 1.050970 & \multicolumn{2}{|c|}{0.9480256} & 0.9487241 \\
\hline & Qd & 0.9977651 & 1.003446 & \multicolumn{2}{|c|}{1.002310} & 9.925960 \\
\hline & Exp & 0.9807432 & 0.9651552 & \multicolumn{2}{|c|}{1.0343102} & 0.9379330 \\
\hline & $\mathrm{C} 4$ & 0.9488392 & 0.7139708 & \multicolumn{2}{|c|}{0.7358081} & 0.3177479 \\
\hline & $\mathrm{C} 2$ & 0.8650733 & 0.9779437 & \multicolumn{2}{|c|}{0.6767941} & 0.4360844 \\
\hline
\end{tabular}

\subsubsection{MSE and MSE Ratio Graphs for Various Sample}

\section{Sizes}

Figure 6 up to figure 10 show graphical representation of relative efficiency for the respective population functions. The MSE ratios for the five functions are seen to mostly concentrated at a point slights below one. This implies that the nonparametric estimator; $\hat{y}_{P S}$ is more efficient than $\hat{y}_{H T}$ and that it's a robust estimator.

\section{Spline vs Horvitz Thompson MSE Ratio}

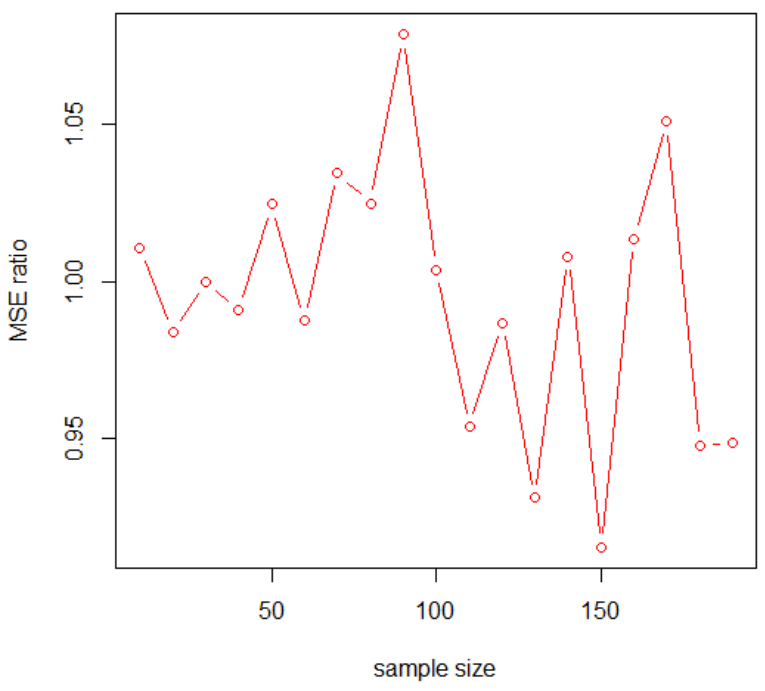

Figure 6. Linear.
Spline vs Horvitz Thompson MSE Ratio

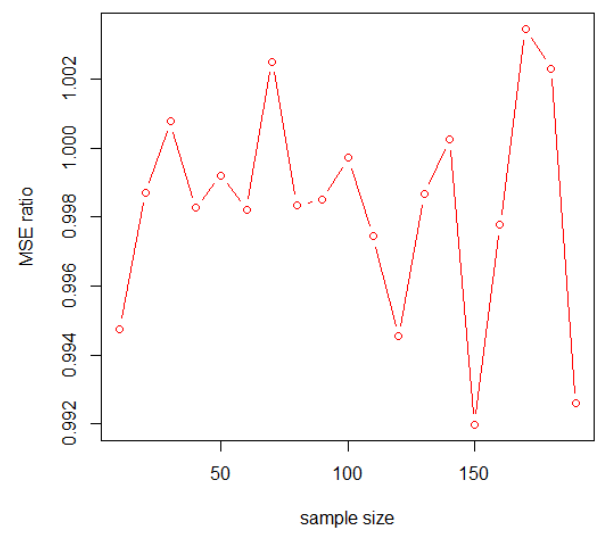

Figure 7. Quadratic.

Spline vs Horvitz Thompson MSE Ratio

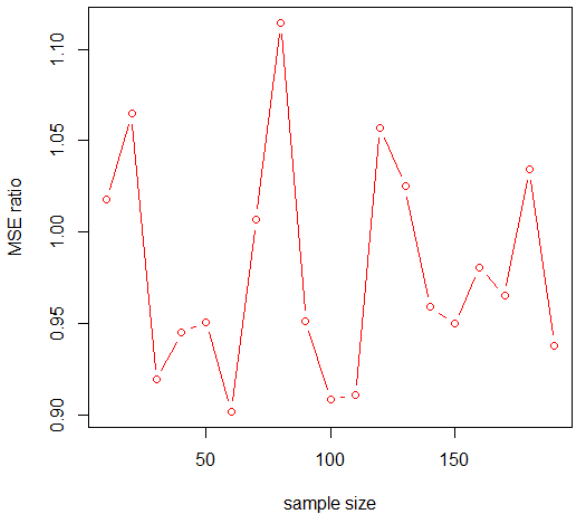

Figure 8. Exponential. 


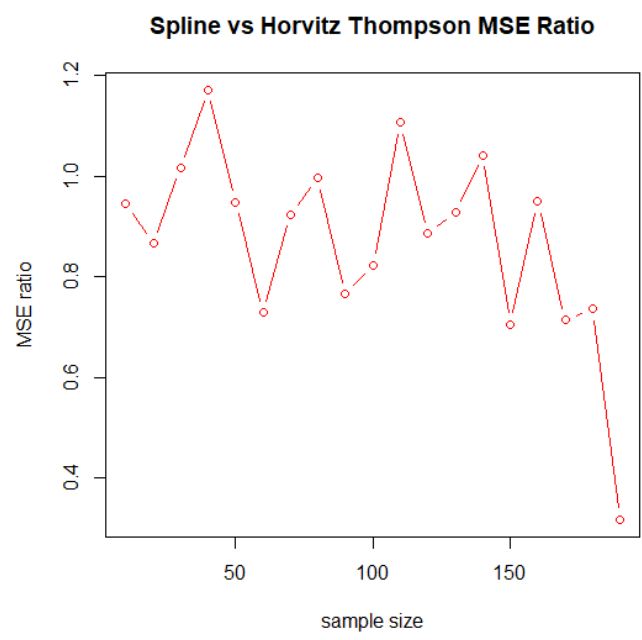

Figure 9. Cycle 4.

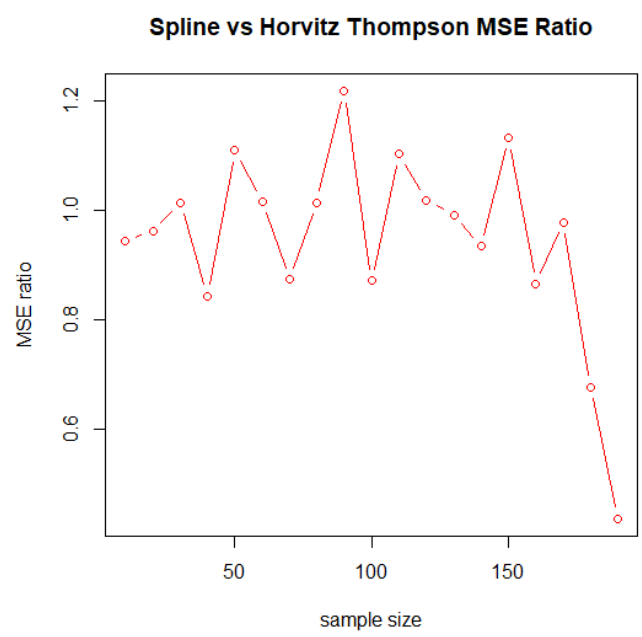

Figure 10. Cycle 2.

\section{Conclusion}

The results from this study show that, the nonparametric calibrated estimator; $\hat{y}_{P S}$ is slightly more efficient than $\hat{y}_{H T}$. The estimator; $\hat{y}_{P S}$ is also very robust because it is not failing for all the five functions compared with the design estimator $\hat{y}_{H T}$ which is known as a well-performing estimator. Generally, the results have also shown that the performance of the nonparametric estimator $\hat{y}_{P S}$ is indistinguishable from that of the design estimator in some instances, and that $\hat{y}_{P S}$ is a normal, unbiased and consistent estimator. Therefore, this study concludes that the nonparametric model calibrated estimators; it is a robust estimator since it does not fail under misspecification.

Due to these good properties of nonparametric model calibrated estimators, this study, therefore, recommends the use of such model calibrated estimators in the estimation of population total in sampling. In the present real-world problem where there are missing variables at both cluster and cluster element levels, yet there is relevant auxiliary information about the variables, model calibrated estimators would be the estimators of choice. This study has further shown that in cases where some cluster and elements within clusters are missing but auxiliary information is available at both levels, then an advantage can be taken of this auxiliary information to fit both cluster totals and cluster elements, which are then calibrated in the estimation of population total. This provides the researcher with a normal, consistent, unbiased, robust and efficient estimator of population total.

\section{References}

[1] Breidt, F. J. and Opsomer, J. D. (2000). Local Polynomial Regression Estimation in Survey Sampling. Annals of Statistics, 28: 1026-1053.

[2] Clair, 1. (2016). Nonparametric kernel estimation methods using Complex survey data, $\mathrm{PhD}$ thesis, momaster university, Main St. West, Hamilton Ontario.

[3] De Boor, C. (2001). A Practical Guide To Splines (Revised Edition). Springer, New York.

[4] Deville, J. C. and Sarndal C. E. (1992). Calibration Estimators in Survey Sampling. Journal of the American Statistical Association, 87: 376-382.

[5] Eilers, P. H. C. and Marx, B. D. (1996). Flexible Smoothing with B-Splines and Penalties (with discussion). Statistical Science, 11: 89-121.

[6] Eubank, R. L. (1988). Spline smoothing and Nonparametric regression. New york and Basel: Marcel Dekker.

[7] Horvitz, D. G and Thompson, D. J. (1952). A Generalization of sampling without Replacement from Finite Universe. Journal of American Statistical Association, 47: 663-685.

[8] Nthiwa Janiffer Mwende et al. (2020). Population Total Estimation in a Complex Survey by Nonparametric Model Calibration Using Penalty Function Method with Auxiliary Information Known at Cluster Levels. American Journal of Theoretical and Applied Statistics. 4: 162-172.

[9] Pinheiro, J. C. and Bates, D. M (2000). Mixed-effects models in S and S-PLUS, Springer: New York.

[10] Rao, S. S. (1984). Optimization Theory and Applications. Wiley Eastern Limited.

[11] Sahar, Z. Z. (2012). Model-based methods for robust finite population inference in the presence of external information. The University of Michigan.

[12] Sayed, A. M. (2010). Nonparametric kernel density estimation using auxiliary information from complex survey data. Masters thesis.

[13] Wahba, G. (1990). A comparison of GCV and GLM for Choosing the Smoothing parameters in the Generalized Spline smoothing problem. Annals of Statistics, 4: 1378-1407.

[14] Wu, C. and Sitter, R. R. (2001). A Model Calibration Approach to Using Complete Auxiliary Information from Survey Data. Journal of American Statistical Association, 96: 185-193.

[15] Zheng and Little (2003): Penalized Spline Model-Based Estimation of the Finite Populations Total Journal of Official Statistics, 19: 99-117. 